\title{
Knowledge-Based Energy Damage Model for Evaluating Industrialised Building Systems (IBS) Occupational Health and Safety (OHS) Risk
}

\author{
Nor Haslinda Abas ${ }^{1, a}$, Nick Blismas ${ }^{2}$ and Helen Lingard ${ }^{2}$ \\ ${ }^{1}$ Faculty of Civil and Environmental Engineering, Universiti Tun Hussein Onn Malaysia, 86400 Parit Raja, Johor, \\ Malaysia \\ ${ }^{2}$ School of Property, Construction and Project Management, RMIT University, Melbourne VIC 3001, Australia
}

\begin{abstract}
Malaysia's construction industry has been long considered hazardous, owing to its poor health and safety record. It is proposed that one of the ways to improve safety and health in the construction industry is through the implementation of 'off-site' systems, commonly termed 'industrialised building systems (IBS)' in Malaysia. This is deemed safer based on the risk concept of reduced exposure, brought about by the reduction in onsite workers; however, no method yet exists for determining the relative safety of various construction methods, including IBS. This study presents a comparative evaluation of the occupational health and safety (OHS) risk presented by different construction approaches, namely IBS and traditional methods. The evaluation involved developing a model based on the concept of 'argumentation theory', which helps construction designers integrate the management of OHS risk into the design process. In addition, an 'energy damage model' was used as an underpinning framework. Development of the model was achieved through three phases, namely Phase I knowledge acquisitaion, Phase II - argument trees mapping, and Phase III - validation of the model. The research revealed that different approaches/methods of construction projects carried a different level of energy damage, depending on how the activities were carried out. A study of the way in which the risks change from one construction process to another shows that there is a difference in the profile of OHS risk between IBS construction and traditional methods. Therefore, whether the option is an IBS or traditional approach, the fundamental idea of the model is to motivate construction designers or decision-makers to address safety in the design process and encourage them to examine carefully the probable OHS risk variables surrounding an action, thus preventing accidents in construction.
\end{abstract}

\section{Introduction}

The Malaysian construction industry plays a significant role in the development and growth of the country's domestic economy, generating further demands for construction activities [1-3]. However, the industry has earned the reputation of being a highly hazardous industry due to its high rates of accidents and fatalities [1-7]. There is therefore an urgent need to improve health and safety performance of Malaysia's construction industry.

\footnotetext{
${ }^{\text {a }}$ Corresponding author : nhaslin@uthm.edu.my
} 
One initiative of the Malaysian government to address occupational health and safety (OHS) in construction is the widespread adoption of Industrialized Building Systems (IBS), commonly termed 'offsite' construction. An IBS approach is commonly deemed to be safer than conventional construction because it changes the nature of the construction process, and requires fewer workers onsite. Claims have been made that IBS, or more specifically offsite construction, can reduce site accidents [8-11]. However, the extent of this impact on safety and health in construction is still unclear, as there are no current systems to comparatively assess OHS risks in different construction processes. McKay [10] has identified the OHS risks of both onsite and the offsite processes, but presents a static assessment that lists hazards of specific processes, rather than comparing the extent of the risks of the various processes. There is therefore a need for a robust dynamic method for comparing different construction processes, such as IBS and traditional approaches, to determine the relative safety and health performance of these processes. This study explores the impact of IBS on construction workers' safety and health by providing a thorough investigation of the activities and associated risks in IBS and traditional construction, with the aim of enabling a comparative evaluation of the OHS risks involved in different construction approaches.

In order to evaluate and compare the OHS risks throughout traditional and IBS construction processes, a structured method incorporating specialist OHS knowledge and guidance is required. An 'argumentation theory model' [12]; as cited in Yearwood and Stranieri [13] building on the work of Cooke et al. [14] is proposed as a method to integrate the management of occupational health and safety risk into the design process. Cooke et al.'s work was developed from structured knowledge in the context of uncertainty and discretionary decision making, involving expert reasoning regarding design impacts on OHS risk represented by 'argument trees' [14]. Their model explored the use of argumentation theory in product design (of buildings), which focused on the implementation of physical aspects of the design. Cooke et al. did not evaluate the design or health and safety characteristics of the construction process itself. This study presents the development of a processcentric model that consists of a series of argument trees for best practice reasoning that can be used by designers or decision makers when examining the OHS risks posed in different construction processes. The argument trees consist of structured knowledge which was developed by focusing on the process involved to build a product in various construction processes. The model provides consideration of product and process design concurrently, thus contributing to the body of knowledge. In addition to Cooke et al.'s model, an 'energy damage model' [15] is used as an underpinning framework for developing the present model. The development of this model suggests options for the decisions that can be made by product and process designers, in such a way as to assess the extent to which their design decisions mitigate the OHS risks associated with various types of damaging energies in construction, and thereby offering a more rigorous relative comparison of OHS risks between IBS and traditional approaches.

This paper serves to outline the development of a knowledge-based energy damage model to assess IBS OHS risk in construction processes at the design stage. This paper expands on an earlier working papers by $[16,17]$, in which the main background of this research is described.

\section{Development Process of the Model}

The risk assessment model developed in this study applied an argumentation framework [14] with an energy damage model [18] as an underpinning framework for developing the model. The concept of argumentation was chosen since it provides the framework for modelling best practice reasoning that can be made by product and process designers, in such a way as to assess the extent to which their design decisions mitigate the OHS risk in construction. Meanwhile, the concept of the energy damage model was chosen due to: i) its unique characteristic which views hazards as a source of potentially damaging energy and risk; and ii), its ability to provide strategies to prevent injury which does not depend on human or behaviour changes. The combination of these concepts thereby offers a more rigorous relative comparison of OHS risks between IBS and traditional approaches. The development process of the model consists of three phases, which are: Phase I - data collection or knowledge 
acquisition; Phase II - development of the argument tree (model); and Phase III - validation of the model.

\subsection{Phase I knowledge acquisition}

Knowledge acquisition involves collecting the data that will underpin the model, which are the construction process, the associated risks and the design aspects which have potential to impact construction risks. Data was collected on on-going construction projects, which used the selected construction approaches in order to observe the process involved and to identify the associated OHS risks. This involved a number of site visits to the IBS manufacturing factory and construction sites. Five sites were visited, of which four were considered to represent IBS/offsite and one represented traditional construction. The detailed studies involved talking to the managerial staff, operatives and supervisors, and also observing and documenting operations. Document analysis techniques were also used to obtain the information regarding 'safe design' aspects generally associated with construction processes. This data source was extracted from OHS guidance material, industrial standards and codes. These were used to provide the design related information during the development of the argument trees.

\subsection{Phase II knowledge processing (argument tree mapping)}

The knowledge acquired from Phase I was compared according to the process involved in selected construction approaches. Within this phase, the risks/hazards associated with the activity were categorized into several damaging energies. Next, the information related to design aspects which have potential to impact upon the damaging energies gathered in secondary sources were used to develop an initial representation of the relevant knowledge in the argument tree.

In developing a model that represents the reasoning for decisions around different construction processes, the same method of modelling as the expert reasoning system in ToolSHeD, was used. Unlike ToolSHeD, the argument trees developed in this study differ in two fundamental ways: i) they seek to model process risks as opposed to product risks, and ii) they are undepinned by an 'energy damage model' to assess OHS risks in the design.

The design factors act as the barriers to prevent such energies from causing injury or damage in energy transfers. The extent of damage depends on the amount of energy deflected by the 'barriers'. The use of 'argument trees' for modeling expert reasoning is capable of providing several OHS risk levels for the activities being carried out, and the OHS risk levels were dependent on the design factors/features selected by designers when they plan to execute a construction project using one particular construction approach. Figure 1 depicts a typical design OHS argument tree for the potential gravitational damaging energy from lifting concrete panels. For example, in the activity of 'loading component onto truck', one of the design factors is 'the weight of the element'. Within this factor, there are several options of the component weight that the designer can choose, and the corresponding values of the options. If the weight of the component (beam) were 0.5 tons, then the relative value would be 3 . This value, together with other values pertinent to the design factors/features within the argument tree (depending on designers' decisions) are then inferred at the end of the argument tree (refer to ' $\mathrm{D}$ ' in Figure 1), which eventually gives a risk level of the activity, according to the design decisions opted by the designers.

As mentioned in the previous paragraph, the design factors act as the barriers that prevent such energies from causing injury or damage in energy transfers. If the activity of lifting the component is to be lifted up to more than 3 meters, the potential damage of gravitational energy, that is, falls of object (component), is likely to be high. In other words, the impact of the damage is higher when compared to the component being lifted to a lower height. This results in the weakening of the barriers. However, the combination of other design factors could balance the magnitude of the OHS risk level, thus improving efficiency of the barriers. The use of argument trees for modelling expert reasoning is also better suited to represent the level of 'how safe is safe enough' regarding the 
designer's decision about the 'barriers' to be used to counter damaging energies during the construction process. This level of decision will determine the value of a risk rating at the 'root' node of an argument tree.

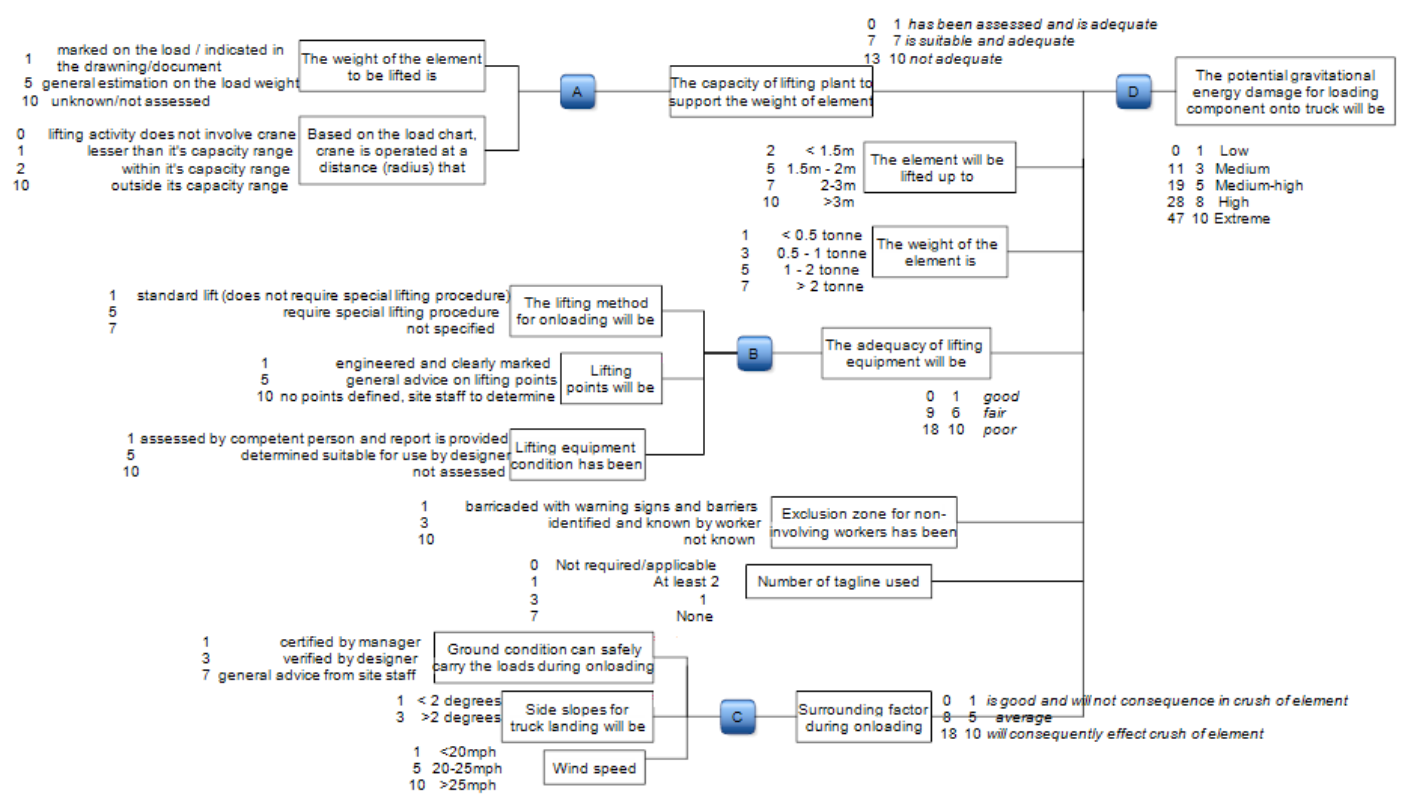

Figure 1. Example of argument tree showing the inference procedure.

As shown in Figure 1 above, the argument tree consists of a set of linguistic values with numerical values, that are assigned to each node of the argument tree, regardless of its position. These values are relevant to the design options available to a designer when making judgement upon aspects of a design, pertinent to the risk of wall cladding construction. The information about the values was sourced from a comprehensive document analysis, such as guidelines and method statements. It can be seen that the tree has linguistic values with corresponding numerical values in the child nodes inferring values to the parent nodes. The numerical values in the tree were initially assigned by the author, which were then modified according to an expert panel's judgement in the validation process. The inference procedure, denoted by $\mathrm{A}, \mathrm{B}, \mathrm{C}$, and $\mathrm{D}$, continues until ultimately inferred at the root node - the final risk rating. The risk rating at the root node indicates either 'extreme', 'high', 'medium' or 'low'. It is measured by calculating the potential of damaging energies which are contingent upon the values decided by the designer at every child node.

\subsection{Phase III validation of the model}

In order to make the model applicable and reliable, this study used scenario testing to validate the model. From the created tool (i.e. the argument tree model), a set of five scenario cases containing knowledge from the trees representing different damaging energies in different construction processes were developed. Each case scenario was assigned with two different scenarios. An expert panel's judgement was obtained to see whether or not their ratings on the scenario case given matches the tool-generated score. In this research, 12 experts were invited to participate. The experts were from various backgrounds and were chosen based on one of the following categories: (i) have experience in contruction projects, or (ii) are responsible for safety in their organisation. They were experienced and credible in their positions and were willing to give commitment to the research project. 
The validation questions consisted of five cases in which each case was assigned with two different scenarios. Given some photographs (for each case), the description of the activities carried out related to the photos and also the scenario information listed within the survey, the respondents were asked to rate the risk of these activities based on the scenarios given. In this close-ended question, respondents were given a Likert scale ranging from 1 (if the respondents think the risk is low) to 10 (if the respondents think the risk is extremely high). The open-ended questions provided an opportunity for respondents to give a reason for their choices using their own words. The comments were useful for the researcher to obtain additional information from respondents' insights that may be overlooked during the development of the argument trees.Once the participants responded to the survey, the responses were analyzed and compared with the results (rating) generated from the tool.

The expert panel's judgement results were compared with those of the tool to determine the consistency of risk ratings between both approaches. Such consistency provides evidence that the knowledge contained in the model is valid. Further, the expert panels' insights on the risk provided valuable information that was adopted in amending the linguistic and numerical values in the argument trees. From the validation process, it was found that the knowledge within the validated argument trees was valid and reliable, involving only minor changes being made in the argument trees.

\section{Demonstration of the Application of the Model}

In order to demonstrate the application of the model, a 'mock trial study' was done, which provides a realistic opportunity to field test the model, and thus visualize the workability of the model. The 'mock' process was done on the existing cases utilised during the model developement. It is acceptable to use the existing cases because these cases were previously used to collect data and information about the process and the risks associated with the process - but not to rate the risk. The information from the case studies was used as if it were the designer's choice of process design arising from discussions made in the 'design' stage. Figure 2 depicts an example of the potential gravitational energy risk profile generated by the tool for the steps of the process involved in the selected construction approaches. The x-axis presents the process steps involved, whereas the y-axis presents the risk magnitude. The lines which plotted the graph represent the selected construction approaches used for this study.

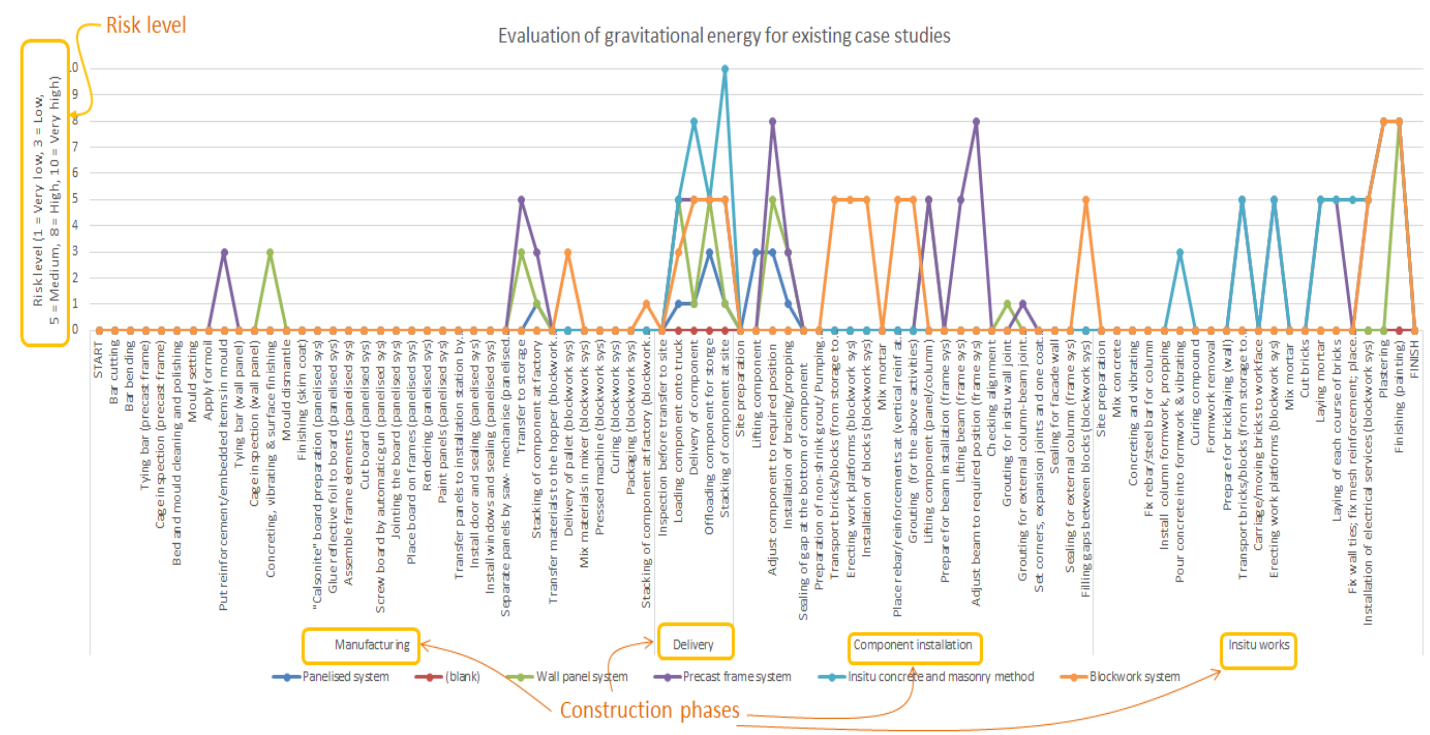

Figure 2. Example of the evaluation of the model for gravitational energy. 
Particulaly noticable are changes to riskover the different steps of the construction processes. This provides evidence that there is a difference in the profile of OHS risk between IBS construction and the traditional method and that these change as work progresses through its component activities. The magnitude of OHS risk for each activity from the damaging energy perspective is also estimated within the tool. The development of this tool demonstrates that it is possible to pick up differences in the risk magnitudes of particular activities between various construction processes. The issue is not that construction approach A is better than B, but rather 'where is the risk?', 'what type of risk is it?', and 'how bad is the risk?'. The tool provides a link between the design and construction phases with regards to construction safety.

\section{Conclusion}

The development process of the argument trees model has been discussed above. The model developed in this study differs from the existing tool in that it is a process-driven model rather than a product-driven model. This is important because product risk is mainly associated with user/operator safety, and not the builder's safety. Process risk however concentrates on the constructor risk, as the constructor is responsible for planning, managing and monitoring the construction process. Using the model, constructors and designers can evaluate the risks in the construction process required for their specified design prior to the construction stage. This could help all parties to formulate a plan for workers' OHS while balancing projects within the planned cost, time and scope.

The use of this model can provide a mechanism for the comparison of hazards of different construction approaches. The tool enables a risk profile to be generated across different phases of a process, thereby providing powerful assistance to designers and contractors. Currently, OHS is considered post-design, and therefore options for hazard elimination are largely removed. This tool offers scenario testing in earlier design phases, so that OHS can inform design, rather than the current approach in which OHS bears little influence over design. Therefore, whether the option is an IBS or traditional approach, the fundamental idea of the model is to motivate construction designers or decision-makers to address safety in the design process and encourage them to examine carefully the probable OHS risk variables surrounding an action, thus preventing accidents in construction.

It is anticipated that the argument tree model will contribute by providing a way for designers to integrate construction process knowledge into design to eliminate or reduce hazards during construction. The fundamental idea will encourage construction designers or decision-makers to address safety in process design and also encourage them to carefully examine the probable OHS risk variables surrounding the construction process.

\section{References}

[1] A.R.A. Hamid, M.Z.A. Majid and B. Singh, An Overview of construction accidents in malaysia," in in issues in construction industry, M.I. Mohamad, Ed., Penerbit Universiti Teknologi Malaysia, Johor Bahru, 110-123, (2008).

[2] S.S. Hosseinian and Z.J. Torghabeh, Major theories of construction accident causation models: A literature review, Int. J. Adv. Eng. Technol., 4(2), 53-66, (2012).

[3] D.N.M.A. Abdullah and G.C.M. Wern, An analysis of accidents statistics in Malaysian construction sector, Proc. of International Conference on E-business, Management and Economics, 3, (2011).

[4] C.L. Foo, Budgeting for occupational safety and health management and its implementation, MBAM Annual Safety Conference, (2005).

[5] SOCSO, Annual Report for 2009, Kuala Lumpur, (2009).

[6] SOCSO, Annual Report for 2000, Kuala Lumpur, (2000).

[7] DOSH, Accident Statistic, Kuala Lumpur, (2013).

[8] A.G.F. Gibb, Off-site Fabrication: Prefabrication, Pre-assembly and Modularisation, Whittles Publishing Services, United Kingdom, (1999). 
[9] T. M. Toole and J. Gambatese, "The trajectories of Prevention through Design in construction.," J. Safety Res., vol. 39, no. 2, pp. 225-30, Jan. 2008.

[10]L. J. McKay, "The Effect of Offsite Construction on Occupational Health and Safety," Loughborough University, 2010.

[11]M. Gangolells, M. Casals, N. Forcada, X. Roca, and A. Fuertes, "Mitigating Construction Safety Risks Using Prevention Through Design.," J. Safety Res., vol. 41, pp. 107-122, 2010.

[12] S. Toulmin, The Uses of Argument, vol. 70. 1958, p. 262.

[13]J. L. Yearwood and A. Stranieri, "The Generic/Actual Argument Model of Practical Reasoning," Decis. Support Syst., vol. 41, no. 2, pp. 358-379, Jan. 2006.

[14]T. Cooke, H. Lingard, N. Blismas, and A. Stranieri, "ToolSHeD TM The Development and Evaluation of a Decision Support Tool for Health and Safety in Construction Design ToolSHeD," Eng. Constr. Archit. Manag., vol. 15, no. 4, pp. 336-351, 2008.

[15]D. Viner, Accident Analysis and Risk Control. New Delhi, India: Viner Pty Ltd., 1991.

[16]N. H. Abas, N. Blismas, and H. Lingard, "Development of a Knowledge-Based Energy Damage Model to Assess Occupational Health and Safety (OHS) Construction Risks in Malaysia," in CIBW099 Prevention: Means to the End of Construction Injuries, illnesses, and Fatalities, 2011.

[17] N. H. Abas, N. Blismas, and H. Lingard, "Knowledge-based energy damage model for evaluating Occupational Health and Safety (OHS) Construction Risks in Malaysia," in HKU-HKHA International Conference 2013 - Construction and Housing in the 21st Century, 2013, pp. 408415.

[18]D. Viner, Accident analysis and risk control. Melbourne: Derek Viner Pty Ltd., 1996. 